\title{
CONTROL DE CABEZA EN NIÑAS CON SÍNDROME DE DOWN POR MEDIO DE LA ESTIMULACIÓN DE LA RESPIRACIÓN, DEL SOPORTE VISCERAL Y DE LA CONEXIÓN CABEZA-COXIS
}

\author{
Carolina Meneses Madrigal, ${ }^{1}$ Ronald Soto Calderón. $^{2}$ \\ ${ }^{1}$ Escuela de Educación Física y Deportes. ${ }^{2}$ Escuela de Educación Especial \\ Universidad de Costa Rica \\ E-mail: carommcr@yahoo.com
}

\begin{abstract}
Resumen
Meneses-Madrigal, C. y Soto-Calderón, R. (2003). Control de cabeza en niñas con Síndrome de Down por medio de la estimulación de la respiración, del soporte visceral y de la conexión cabeza-coxis. Revista en Ciencias del Ejercicio y la Salud, 3(1), 27-34. En esta investigación se aplicó un programa para estimular la respiración, el soporte visceral y la conexión cabeza-coxis en 2 niñas portadoras del Síndrome de Down, con el objetivo de mejorar su control de cabeza. Las niñas participantes tenían uno y cuatro meses de edad. Sus madres fueron entrenadas en la medición de la variable dependiente (levantar la cabeza) y en la aplicación del programa el cual constó de 90 sesiones. La variable dependiente se operacionalizó y se midió en términos de tiempo. Durante 4 sesiones las madres fueron supervisadas en la aplicación del programa. Se aplicó un diseño de sujeto único de múltiples AB. Previo a la aplicación del programa se destinaron 3 mediciones para obtener los puntajes de línea base y así se determinó el nivel operante de la variable dependiente en las niñas. La información recolectada se analizó mediante estadística descriptiva. En este estudio se logró incrementar el tiempo en que las participantes mantenían levantada la cabeza mediante la aplicación de actividades que estimularon funciones y estructuras corporales tradicionalmente no estimuladas en niños con Síndrome de Down. PALABRAS CLAVES: Estimulación temprana, Síndrome de Down, discapacidad, control de cabeza, respiración, soporte visceral, conexión cabeza-coxis.
\end{abstract}

\section{INTRODUCCION}

El movimiento juega un papel preponderante en el desarrollo del ser humano; desde antes de nacer el niño se mueve y estas primeras experiencias de movimiento en los niños son el cimiento sobre el que descansa el desarrollo de su personalidad y de todo su potencial. El movimiento es la primera forma de comunicación con que cuenta el ser humano y es la base de su desarrollo cognoscitivo (Newman y Newman, 1991).

La capacidad de movimiento de un niño determina su comportamiento y el de las personas que lo rodean, por tanto, un niño que tiene limitadas sus posibilidades de movimiento obtiene menos refuerzo de parte de sus cuidadores y se ve privado en la cantidad y calidad de interacción que las personas mantienen con él. "Los retrasos en las adquisiciones motoras tendrán influencia sobre las experiencias del niño y sobre el desarrollo de las otras áreas" (Perera, 1995, p. 79).

El desarrollo motor se da en dirección céfalo-caudal y próximo-distal, por lo cual uno de los primeros logros que se alcanza es el control de cabeza (Newman y Newman, 1991). Es decir, el niño llega a tener dominio de la posición y de los movimientos de su cabeza; esto se da paulatinamente; durante el primer mes de vida, en posición prono, el niño mueve la cabeza hacia los lados y la levanta por breves segundos, a los 2 meses el niño levanta su cabeza de forma intermitente, a los 3 meses logra mantenerla por más tiempo levantada elevando además el pecho de la superficie en que está acostado y a los 4 meses utiliza sus brazos y manos como 
soporte para mantenerla firmemente levantada (Ministerio de Salud, 1987). Niños sin alteraciones en su desarrollo llegan a alcanzar esta destreza a veces antes de las edades aquí mencionadas. El control de cabeza es la base de las destrezas motoras subsiguientes, al alcanzarlo, el niño logrará el control de tronco y de extremidades y desarrollará movimientos de locomoción. El control de cabeza depende de la integridad del sistema nervioso central y del sistema neuro-músculo-esquelético, de la maduración neurológica y de la integración de los reflejos primitivos además de los estímulos ambientales (Calderón, 2000).

El Síndrome de Down es una condición que causa retraso en todas las áreas incluyendo el área motora gruesa, este retraso se debe a la hipotonía muscular y a otras posibles alteraciones del sistema nervioso central que presentan los niños con dicho síndrome (Perera, 1995). El retardo mental que los caracteriza, también los limita en sus experiencias motoras. En ellos el control de cabeza se presenta aproximadamente entre los 6 y 8 meses cuando se les estimula (Meneses, 2000) y es la destreza motora que recibe más atención en sus primeros meses de vida generando además gran preocupación en los encargados.

Por muchos años se han aplicado tratamientos a base de químicos, vitaminas, enzimas y minerales en estos niños buscando disminuir su flacidez muscular; uno de los tratamientos más populares fue el tratamiento con 5 Hidroxitriptofano conocido como 5-HTP. Con este se buscaba aumentar los niveles de serotonina que presentan y que son la causa de su hipotonía. Sin embargo, las investigaciones concluyeron que no fue efectivo para mejorar el tono muscular y que además tenía efectos secundarios dañinos (Rynders y Horrobin, 1986). Romano (1998) realizó una exhaustiva revisión de las diversas terapias médicas que se han aplicado a personas con Síndrome de Down buscando mejoría motora y llegó a la conclusión de que la atención temprana en las diversas áreas del desarrollo (incluyendo la motora gruesa) es esencial para mejorar su calidad de vida y que no basta con las terapias médicas, las cuales, en lo que a mejoría motora específicamente se refiere, no han mostrado resultados importantes. Por todo lo anterior y tomando en cuenta la importancia del desarrollo motor, se hace necesaria la intervención temprana con programas de estimulación que atiendan al niño con Síndrome de Down y a su familia de forma integral cubriendo desde luego la estimulación motora.

La estimulación motora de los niños con Síndrome de Down tradicionalmente ha estado a cargo de fisioterapeutas quienes para mejorar el control de cabeza, básicamente lo que realizan es estimulación neuromuscular obteniendo así la respuesta voluntaria del niño de mantener erguida su cabeza. Sin embargo, en la literatura no se encuentran investigaciones al respecto en donde se haya sistematizado de forma controlada algún tratamiento con este fin. En este estudio se proponen actividades que no contradicen el trabajo realizado por fisioterapeutas pero que abarcan estructuras y funciones corporales que en fisioterapia no se estimulan directamente tales como la respiración, el soporte visceral y la conexión cabeza-coxis, esto con el objetivo de involucrarlas activamente en la acción de levantar la cabeza ya que como lo expresa Bainbridge (1993), cuando se dirige la atención a diferentes áreas del cuerpo para iniciar el movimiento desde ellas, la cualidad del movimiento cambia. El enfoque del movimiento que propone esta autora va más allá del modelo biomecánico tradicional pues reconoce la gran movilidad que tienen otras estructuras corporales además del sistema músculo-esquelético y su importante papel en la producción del movimiento. Dicha autora ha aplicado este enfoque en un amplio rango de personas que incluye desde habilidosos bailarines y atletas hasta adultos mayores y personas con dificultades motoras y principalmente niños con disfunciones neurológicas (Johnson, 1997). 
Basándose en el trabajo de Bainbridge (1993), Grubinger (2000) realizó un estudio de caso con un niño de año y nueve meses diagnosticado con retraso motor. Este niño, nacido en un parto difícil, presentaba problemas de salud desde su nacimiento, sus movimientos eran desordenados, tenía bajo tono muscular y pobre registro de sensaciones. El tratamiento al que fue sometido abarcó 10 sesiones durante 6 meses en las que se le estimuló el tono de los órganos y los músculos, el sentido táctil y las funciones simpática y parasimpática, logrando mejorías importantes en sus patrones de movimiento los cuales se tornaron más organizados.

Siguiendo este mismo enfoque, el programa de intervención que se aplica en esta investigación, busca compensar la flacidez muscular de los niños con Síndrome de Down por medio de la estimulación de otras estructuras y funciones corporales que intervienen en el movimiento, ya que por su marcada flacidez, estos niños generan muy poca fuerza muscular. Las funciones y estructuras que se estimulan en el presente estudio se describen a continuación según Bainbridge (1993):

1. Respiración: la función respiratoria es un movimiento interno que subyace y sirve de soporte al movimiento del cuerpo en el espacio ya que da volumen internamente a la estructura músculo-esquelética.

2. Soporte visceral: los órganos o vísceras también dan soporte a la estructura músculo-esquelética, cuando hay bajo tono visceral, se presenta bajo tono muscular y desorganización en el esqueleto, es decir, el tono de los órganos está íntimamente relacionado con el tono postural básico.

3. Conexión cabeza-coxis: el sistema óseo y dentro de él la conexión cabeza-coxis, constituye la estructura básica de sostén que permite levantar el peso en relación con la gravedad por medio del adecuado establecimiento de líneas de fuerza a través del esqueleto.
Cada uno de estos sistemas hace su propia contribución al movimiento pero a su vez son interdependientes.

La presente investigación propone un programa de estimulación para incrementar el tiempo en que niños pequeños con Síndrome de Down mantienen levantada su cabeza, este programa compensa su flacidez muscular pues se centra en otras estructuras corporales, lo cual es apoyado por Gibson y Harris (1988, p.86) quienes afirman que "los niños con Síndrome de Down no son simplemente retrasados sino que tienen una serie de dificultades específicas de aprendizaje y los programas de intervención tienen que estructurarse intentando compensar directamente esas dificultades". La presente propuesta es además novedosa y pionera en su campo y ofrece una nueva opción para la estimulación motriz de los niños con Síndrome de Down, con todas las implicaciones positivas que esto conlleva en las demás áreas del desarrollo, pues como ya se mencionó al inicio, el desarrollo motor es el cimiento sobre el que descansa el desarrollo de la personalidad y de todo el potencial del niño.

\section{METODOLOGIA}

\section{Diseño}

Se aplicó un diseño de sujeto único en su variante de múltiples $A B$, la cual fue propuesta por el investigador Fonseca según cita Soto (1994) y ha sido aplicada con éxito por diversos investigadores en estudios con niños con necesidades especiales, entre ellos Cabezas y Fonseca, (1992 a y b) y Soto, (1994). Este diseño con esta variante específica, permitió el análisis de los efectos directos de un único procedimiento de intervención en cada participante.

En esta investigación se obtuvieron múltiples $\mathrm{AB}$, es decir, a cada participante se le midió el nivel operante de la variable dependiente (línea base) y el nivel de respuesta de dicha variable cuando fue sometida al tratamiento (intervención), esto 
permitió realizar un análisis no sólo intrasujeto sino también trans-sujeto y así se conoció la consistencia de los resultados entre niños y en cada uno de ellos.

\section{Participantes}

Participaron en este estudio 2 niñas con Síndrome de Down que no alcanzaban un control de cabeza superior a los tres minutos, sus características se resumen a continuación:

a. Participante M: niña de un mes exacto de nacida al iniciar el tratamiento, mostró respuestas visual y auditiva acordes con su edad $y$ un tono muscular evidentemente bajo además de conducta motora muy pasiva, al nacer se le diagnosticó una cardiopatía. M no había recibido ningún tipo de estimulación antes de que se incluyera en este estudio.

b. Participante $\mathrm{C}$ : niña de tres meses y tres días de nacida al iniciar el tratamiento con respuestas visuales y auditivas adecuadas y con mucha actividad motriz. Esta niña tenía mes y medio de recibir estimulación temprana en un centro de educación especial, sin embargo dicho tratamiento fue suspendido mientras participó en este estudio.

Por medio de un contrato se obtuvo el consentimiento respectivo por parte de los encargados para la participación en el estudio de cada uno de las niñas.

\section{Registro de la información}

Se utilizó un registro de duración (Mayer y Sulzer, 1990) para determinar el tiempo en que los participantes mantuvieron la cabeza levantada. La variable dependiente corresponde a levantar la cabeza y se definió como la acción de mover la cabeza hasta despegar la cara de la superficie en que están acostadas en posición prono. Las mediciones se realizaron con la niña en posición prono y con sus brazos y manos hacia el frente. Estas mediciones se realizaron en el periodo de línea base y en el periodo de intervención al finalizar cada sesión de tratamiento.
Las madres de los niños fueron entrenadas para registrar la información.

\section{Procedimientos}

Se destinaron 3 mediciones en días distintos para línea base, estas mediciones se realizaron siguiendo el mismo procedimiento que se aplicó para las mediciones de la fase de intervención el cual se describe más adelante. El periodo de intervención tuvo una duración de 30 días, durante los cuales se aplicó el programa diariamente tres veces en horas fijas para cada niña hasta donde fue posible, para un total de noventa sesiones con cada una de ellas. Luego de cada una de esas sesiones de trabajo, se realizó la respectiva medición obteniendo así noventa mediciones procedentes del proceso de intervención.

Posteriormente, se entrenó a cada madre de forma individual en la aplicación del programa y en el registro de la información, hasta tener seguridad de que dominaba todo lo relacionado con el programa y la medición. Durante estas sesiones de entrenamiento que se realizaron en la casa de cada niña, se conoció el ambiente en el que ellas viven y se hicieron los ajustes necesarios en espacio físico y otros aspectos, para así lograr una adecuada aplicación del programa.

El programa con las actividades para mejorar el control de cabeza se entregó a las madres por escrito en un documento de fácil comprensión, este fue revisado por una educadora somática, dos fisioterapeutas y un médico; quienes descartaron riesgos $\mathrm{o}$ contraindicaciones del mismo; se realizaron además varias aplicaciones piloto del programa con madres ajenas a este estudio para descartar problemas relacionados con la comprensión de las instrucciones. El programa además se les entregó grabado en un cassette con el objetivo de que al aplicarlo no necesitaran leerlo sino que lo escucharan, de esta forma se logró uniformidad en la duración de cada actividad entre sesiones en un mismo niño y entre niños, pues la 
grabación indica en qué momento empezar y terminar cada actividad.

El programa consta de tres partes, la primera parte contiene actividades para estimular la respiración (presión y golpeteo suaves a nivel toráxico), la segunda para estimular el soporte visceral: (vibración producida con la voz), y la tercera para estimular la conexión cabeza-coxis (movimientos de cabeza y tacto en columna vertebral); las actividades en total tienen una duración de 15 minutos

Al finalizar las actividades, las madres colocaron a las niñas boca abajo por tres minutos y contabilizaron los segundos en que levantaron la cabeza según la definición dada para esta variable en el apartado anterior. Durante la medición las madres sonaron un juguete frente a las niñas para propiciar la conducta, cada vez que las niñas levantaban la cabeza, detenían el sonido y le acercaban el juguete a la vista, cuando las niñas bajaban la cabeza, sus madres volvían a producir el sonido. Se utilizó como juguete el sonajero preferido de cada niña, este juguete era de uso exclusivo durante las mediciones mientras duró la investigación. Para facilitar esta medición, la grabación contó con tres minutos en los que se marcaba cada segundo con un sonido, de modo que las madres no necesitaban ver el reloj sino que contaban los segundos por medio del sonido.

\section{Análisis estadístico}

El análisis estadístico se realizó mediante estadística descriptiva, se analizaron los promedios y el comportamiento de las curvas para cada participante, tomando en cuenta las características del diseño de sujeto único.

\section{RESULTADOS}

En el cuadro $\mathrm{N}^{\mathrm{o}} 1$ se muestra el tiempo promedio total en que cada una de las participantes levantó la cabeza en la fase de línea base y de intervención.

Se observa claramente un cambio en el nivel de respuesta de cada niña entre una fase y la otra. En el cuadro $\mathrm{N}^{0} 2$ se detalla esta información. En él se presentan los promedios semanales para cada participante; la madre de $\mathrm{M}$ continuó voluntariamente las sesiones así que esta niña se sometió al tratamiento durante 6 semanas mientras que C durante 4 semanas.

Cuadro 1. Tiempo promedio total en segundos de control de cabeza

\begin{tabular}{lcc}
\hline & \multicolumn{2}{c}{ Niñas } \\
\cline { 2 - 3 } & $\mathbf{M}$ & $\mathbf{C}$ \\
\cline { 2 - 3 } Línea Base & $0.33 \mathrm{~s}$ & $25.66 \mathrm{~s}$ \\
Intervención & $6.64 \mathrm{~s}$ & $137.74 \mathrm{~s}$ \\
\hline
\end{tabular}

Cuadro $\mathrm{N}^{0} 2$. Tiempo promedio semanal de control de cabeza

\begin{tabular}{lcc}
\hline & \multicolumn{2}{c}{ Niñas } \\
\cline { 2 - 3 } & $\mathbf{M}$ & $\mathbf{C}$ \\
\hline Línea Base & $0.33 \mathrm{~s}$ & $25.66 \mathrm{~s}$ \\
Semana 1 & $5.46 \mathrm{~s}$ & $85.36 \mathrm{~s}$ \\
Semana 2 & $6.85 \mathrm{~s}$ & $156.19 \mathrm{~s}$ \\
Semana 3 & $5.91 \mathrm{~s}$ & $161.66 \mathrm{~s}$ \\
Semana 4 & $6.18 \mathrm{~s}$ & $157.44 \mathrm{~s}$ \\
Semana 5 & $7.6 \mathrm{~s}$ & - \\
Semana 6 & $9 \mathrm{~s}$ & - \\
\hline
\end{tabular}

Al graficar los valores de $\mathrm{M}$ (ver gráfico No1) se visualiza claramente el bajo rendimiento en la conducta en estudio antes de ser intervenida, así como la progresiva mejoría una vez iniciada la intervención, manteniéndose una tendencia ascendente en las últimas sesiones.

En el gráfico No2 correspondiente a C, la curva asciende al iniciar la intervención, para luego mostrar un comportamiento más estable, pero siempre superior al de la línea base.

\section{DISCUSION}

El programa propuesto en esta investigación propició un incremento en el tiempo en que las niñas levantaban su cabeza al comparar el periodo de línea base con el de 
intervención, esto se logró por medio de la estimulación de estructuras y funciones corporales que intervienen en el movimiento tales como la función respiratoria, el soporte visceral y la conexión cabeza-coxis (Bainbridge, 1993).

\section{Gráfico $\mathbf{N}^{\mathbf{0}} 1$}

Tiempo de control de cabeza de M

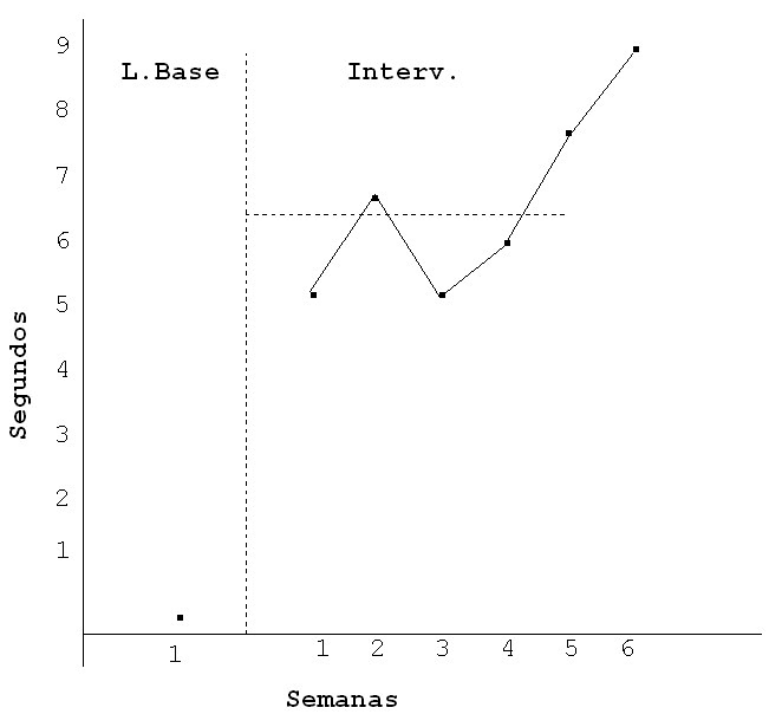

Gráfico $\mathbf{N}^{0} 2$

Tiempo de control de cabeza de $\mathrm{C}$

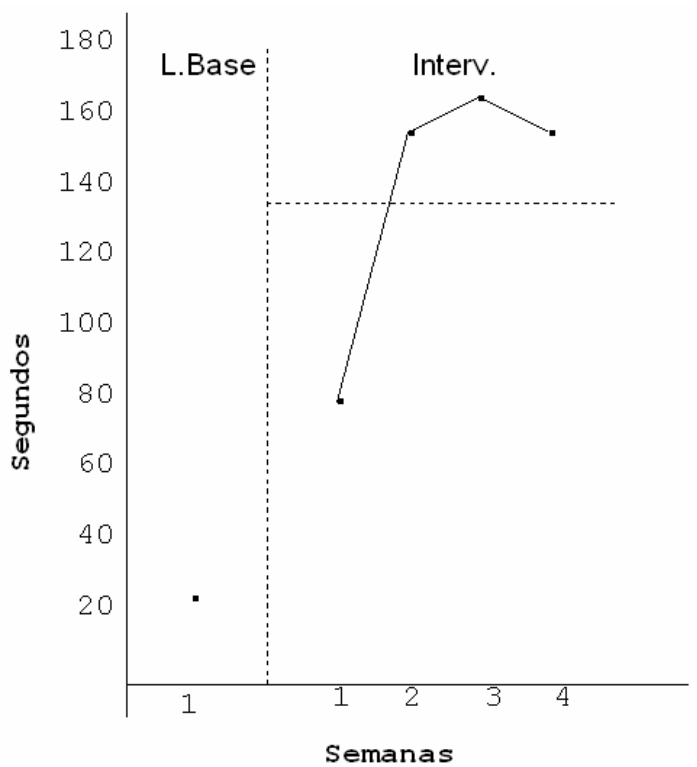

Antes de ser intervenida la conducta, M tenía un nivel de respuesta promedio inferior a un segundo $(0.33 \mathrm{~s})$, al iniciar el tratamiento y desde la primera semana, el promedio de respuesta aumentó. Aunque la propuesta original de este estudio era de 4 semanas de tratamiento, la madre de M continuó voluntariamente con él, lo que permitió confirmar la tendencia ascendente de la conducta, ya que en las primeras 4 semanas la respuesta mostró un comportamiento fluctuante. Se debe tomar en cuenta también que en la cuarta semana $M$ ya tenía 2 meses de vida, es decir estaba más madura y había sido expuesta por más tiempo al tratamiento y a la posición prono. Las mediciones realizadas no permitieron determinar los intervalos en los que se distribuían los segundos registrados, sin embargo ya sea de forma consecutiva o aislada, cada uno de esos segundos tiene gran valor para el desarrollo motor de $\mathrm{M}$ quien alcanzó un promedio de 6.64 segundos al cabo de 6 semanas de tratamiento.

En el caso de C, la línea base mostró un promedio cercano al medio minuto (25.6 s). Durante las 3 primeras semanas de intervención la curva de respuesta ascendió, en la cuarta semana se observó un leve descenso el cual no fue inferior a los valores de las semanas anteriores. El tiempo promedio de control de cabeza durante las 4 semanas fue de 137.74 segundos, es decir 2 minutos y 17.4 segundos. En varias de las sesiones C mantuvo la cabeza levantada durante los 3 minutos de medición. Debido a que las mediciones se programaron para cuatro semanas, con esta participante no se pudo conocer el comportamiento de la curva después del mes de tratamiento.

La mejoría alcanzada en ambas niñas favoreció su desarrollo en general ya que, como se mencionó al inicio, el movimiento juega un papel preponderante en el desarrollo de la personalidad y de todo el potencial del ser humano. Cuando un niño que está en posición prono mueve su cabeza hasta despegar su cara de la superficie en que está acostado, recibe mayor información visual y 
tiene más posibilidades de contacto social, este logro a su vez motiva al cuidador para seguir reforzando esta conducta la cual se ve fortalecida. Además, los movimientos de cabeza en los primeros meses de vida aportan importante información a los sentidos propioceptivo y vestibular, los cuales son fundamentales en el desarrollo motor. El control de cabeza es la base de destrezas motoras posteriores como control de tronco y de extremidades, entre otras (Calderón, 2000).

El programa aplicado tiene la ventaja de que no expone al niño a movilizaciones bruscas ni repeticiones aburridas tanto para él como para la persona que le está estimulando; si se realiza correctamente, siguiendo las instrucciones, propicia además un espacio para fortalecer el vínculo afectivo entre el niño y su cuidador y puede traer beneficios como regulación de la función digestiva ya que algunos niños con Síndrome de Down sufren de estreñimiento (Saborío, 2000), aumento en la emisión de sonidos vocálicos y fortalecimiento de músculos encargados de la respiración; estos aspectos no se registraron en este estudio por lo cual resulta valioso tomarlos en cuenta para una futura investigación.

Igualmente importante sería registrar las cualidades del movimiento, ya que aunque no era un objetivo de este estudio, se observó en las niñas mayor estabilidad a la hora de levantar la cabeza, de esta forma un movimiento de cabeza tambaleante que se presentaba al inicio, disminuyó en $\mathrm{M}$ y desapareció en C; según Bainbridge (1993), una posible explicación para esto es la mayor participación de los órganos en la acción de elevar la cabeza.

Durante las semanas en que se aplicaron los tratamientos, se presentaron factores como citas médicas, cambios en el horario de sueño y problemas de salud, debido a ellos las madres no siempre podían cumplir con las tres sesiones diarias por tanto, aunque $\mathrm{M}$ fue sometida a dos semanas más de tratamiento, ambas niñas recibieron un número similar de sesiones: M 70 sesiones y C 67 sesiones. Es decir, el tratamiento de $\mathrm{C}$ fue más intensivo que el de M. Esto podría ser una causa de que $\mathrm{C}$ tuviese mejores resultados, otra causa podría ser la diferencia de edad entre las niñas, además hay que tomar en cuenta que $\mathrm{M}$ también estaba en desventaja por su cardiopatía y porque nunca había participado en sesiones de estimulación temprana mientras que $\mathrm{C}$ sí. Esto se evidenció desde un inicio donde se computaron los promedios de sus líneas base, los cuales eran diferentes.

Como se ha visto hasta el momento, son muchas las variables que interactuaron con cada una de las niñas; el diseño de sujeto único de múltiples $\mathrm{AB}$, aplicado en este estudio, no pretende controlar esos factores sino que los aprovecha como fuente de información estudiando cuidadosamente cada caso de forma aislada.

$\mathrm{Si}$ en cada uno de los participantes (a pesar de la variabilidad de factores) se presentan efectos similares ante el procedimiento de intervención, se puede afirmar que dichos efectos se deben al tratamiento que tuvieron en común tal y como sucedió en el presente estudio (Fonseca, 2000) Si bien es cierto, el diseño aplicado no permite generalizaciones de los resultados, sí resulta valioso como base para estudios futuros sobre la estimulación motriz temprana.

Está de más mencionar la importancia de continuar investigando sobre la estimulación motora en niños con Síndrome de Down y niños con otras alteraciones en el desarrollo. Resulta arriesgado aplicar tratamientos cuyos efectos no hayan sido estudiados de forma sistemática por lo que el presente estudio aporta valiosa información para el planteamiento de nuevas preguntas de investigación, que enriquezcan las opciones terapeúticas y de estimulación en esta población.

\section{REFERENCIAS}

Bainbridge, B. (1993). Sensing Feeling and Action. Massachusetts: Contact Editions.

Cabezas, H. y Fonseca, G. (1992a). Entrenamiento en la adquisición de la imitación verbal en 3 niños con autismo. Revista Educación, 16(1), 101-106. 
Cabezas, H. y Fonseca, G. (1992b). Uso del condicionamiento operante para la adquisición del contacto ocular en cuatro niños con autismo. Revista Educación, 16(2), 19-23.

Calderón, M. (2000). Entrevista personal realizada en marzo 2000 a Maritza Calderón, Fisioterapeuta del Centro San Felipe Neri. San José, Costa Rica.

Fonseca, (2000). Entrevista personal realizada en febrero 2000 al Psicólogo Gerardo Fonseca. San José, Costa Rica.

Gibson, D. y Harris, A. (1988). Agregated early intervention effects for Down's syndrome persons: Patterning and longevity of benefits. Journal of Ment. Defic. Res., 32, 1-17.

Grubinger, L. (2000).Twelve visits to a BMC Playroom. Currents, winter-spring, 7-17

Johnson, D. (1997). Groundworks Narratives of embodiment. California: North Atlantic Books.

Mayer, R. y Sulzer, B. (1990). Procedimientos del análisis conductual aplicado con niños $y$ jóvenes. México: Editorial Trillas.
Ministerio de Salud. (1987). Escala para la Evaluación del Desarrollo Integral del Niño de cero a seis años. Costa Rica: Departamento de Salud Mental.

Newman, B. y Newman, P. (1991). Desarrollo del Niño. México: Editorial Limusa.

Perera, J. (1995). Síndrome de Down: aspectos específicos. Asociación Europea para el síndrome de Down.

Romano, C. (1998). Review of the controversial medical therapies in Down's Syndrome. European Down's Syndrome Association News Letter, 10, 4-6.

Rynders, J., y Horrobin J. (1986). A guide for new parents of children with Down's Syndrome. University of Minnesota.

Saborío, M.. (2000). Entrevista personal realizada en mayo 2000 al Dr. Manuel Saborío. Hospital Nacional de Niños. San José, Costa Rica.

Soto, R. (1994). Un sistema alternativo en la comunicación de niños con autismo. Universidad de Costa Rica. 